Trauma Berufskrankh 2010 · 12[Suppl 3]:329-334 DOI 10.1007/s10039-009-1569-z

Online publiziert: 10. Januar 2010

(c) Springer-Verlag 2010

D. Schneidmueller ${ }^{1} \cdot$ M. Maier ${ }^{1} \cdot$ M. Mack ${ }^{2} \cdot$ E. Geiger $^{1} \cdot$ I. Marzi ${ }^{1}$

${ }^{1}$ Abteilung für Unfall-, Hand- und Wiederherstellungschirurgie, Johann-Wolfgang-Goethe-Universität Frankfurt, Frankfurt am Main

${ }^{2}$ Abteilung für diagnostische und interventionelle Radiologie, Johann-Wolfgang-Goethe-Universität Frankfurt, Frankfurt am Main

\title{
Gelenkverletzungen im Kindes- und Jugendalter
}

\section{Indikation und therapeutische Relevanz der MRT (Magnetresonanztomographie)}

nahmetechnik bei frischen Verletzungen schmerzbedingt nicht möglich ist. Daneben bietet das wachsende Skelett einige Besonderheiten in der Röntgendiagnostik, wie noch nicht ossifizierte Knochenanteile, zusätzliche Ossifikationszentren und Wachstumsfugen, die bei der Beurteilung Schwierigkeiten bereiten können und einer großen Erfahrung bedürfen. Somit werden immer wieder Frakturen in der primären Röntgendiagnostik übersehen, v. a. an komplexen Gelenken wie dem Ellenbogengelenk [12, 25, 29, 30]. Aus diesem Grund wird die MRT von vielen Autoren als weiterführende Bildgebung in unklaren Fällen empfohlen $[3,5$, $14,15,16,23,24]$.

Anhand einer eigenen Untersuchung und einer Literaturübersicht sollen die Wertigkeit der MRT für Gelenkverletzungen im Kindes- und Jugendalter untersucht sowie eine Übersicht über die unterschiedlichen Indikationen an der jeweiligen Lokalisation gegeben werden.

\section{Material und Methode}

In einer retrospektiven Analyse wurden alle Kinder ( $\leq 16$. Lebensjahr) erfasst, bei denen in den letzten 3 Jahren nach konventioneller Röntgendiagnostik eine MRT in der Abteilung für diagnostische und interventionelle Radiologie der JohannWolfgang-Goethe-Universität Frankfurt durchgeführt wurde. Ausgeschlossen wurden Patienten, bei denen die Wachstumsfugen bereits geschlossen waren, ein primäres konventionelles Röntgenbild nicht vorlag, kein akutes Trauma oder ein Tumorleiden in der Anamnese vorhanden oder bereits Voroperationen erfolgt waren.

Ausgewertet wurden die Röntgen- sowie MRT-Bilder unabhängig voneinander, verblindet von 2 Radiologen und 2 Unfallchirurgen, nach einem hierfür entwickelten standardisierten Fragebogen. Die endgültige Diagnose wurde im Konsens gestellt.

Die Fraktureinteilung erfolgte im Kindesalter nach Salter u. Harris bzw. bei den Übergangsfrakturen in "twoplane“- oder „triplane“-Frakturen. Anhand der gestellten Diagnose in den Röntgen- und den MRT-Untersuchungen wurde von 2 Unfallchirurgen jeweils retrospektiv eine Therapieempfehlung gegeben und diese später miteinander verglichen.

\section{Ergebnisse und Diskussion}

Gelenkfrakturen im Kindesalter sowie das genaue Ausmaß der Frakturdislokation sind häufig im konventionellen Röntgenbild nicht exakt zu diagnostizieren. Belassene Stufen im Gelenkbereich erhöhen wie beim Erwachsenen das Risiko einer posttraumatischen Arthrose und 

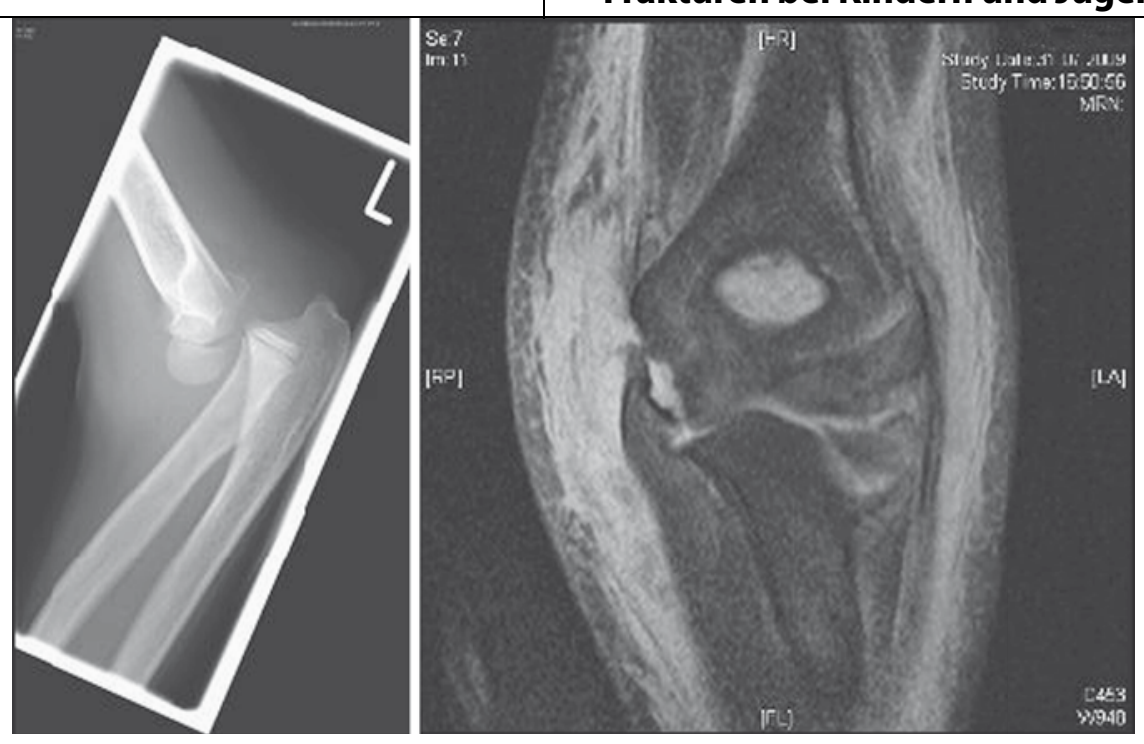

Abb. 1 \ Zustand nach Ellenbogenluxation mit knöchernem Seitenbandausriss

sollten nicht belassen werden. Durch die unmittelbare Nähe der Wachstumsfuge kann es nach Traumen in Gelenknähe zu Wachstumsstörungen der betroffenen Extremität kommen. Das Ausmaß der Wachstumsstörung ist abhängig vom Alter des Kindes, von der Lokalisation sowie dem Dislokationsausmaß der Fraktur und der Anzahl der Repositionsversuche. Somit sind für eine differenzierte endgültige Therapie eine primäre Diagnose und Klassifikation der Verletzung in Kenntnis des genauen Frakturverlaufs notwendig.

Gerade bei fraglichen oder okkulten kindlichen Frakturen hat sich die MRT im Vergleich zur konventionellen Röntgendiagnostik in der Vergangenheit bewährt und wird häufig als erweiterte Diagnostik in unklaren Fällen empfohlen [21]. Jaramillo et al. [15] konnten anhand von 28 MRT-Untersuchungen an verschiedenen Lokalisationen in 6 Fällen eine Änderung der Frakturklassifikation durch die MRT beobachten. Carey et al. [5] analysierten 14 MRT-Aufnahmen und erhielten in 50\% der Fälle eine Änderung der Diagnose, welche in $36 \%$ der Fälle zu einer Veränderung des therapeutischen Vorgehens führte. Von Zitzewitz et al. [34] beobachteten eine Rate von 38\% zusätzlich diagnostizierten Frakturen in der MRT.

Insgesamt konnten 45 Gelenke - 6 Ellenbogen-, 18 Knie- und 21 Sprunggelenke - ausgewertet werden.

\section{Ellenbogen}

Im eigenen Krankengut resultierte in 2 der 6 Fälle eine Änderung der Diagnose aus der MRT-Untersuchung. In 2 Fällen konnte eine begleitende Bandverletzung sowie in 5 Fällen ein "bone bruise“ dargestellt werden. In keinem der Fälle änderte sich das therapeutische Vorgehen durch die gewonnenen Zusatzinformationen.

Aufgrund der Komplexität des Gelenks und der noch nicht ossifizierten Bereiche wird die MRT häufig als weiterführende Methode empfohlen [3, 10, 24, 32]. Bezüglich der therapeutischen Konsequenz jedoch gibt es unterschiedliche Beobachtungen in der Literatur. Einige Autoren konnten eine Änderung des Vorgehens aus den Zusatzinformationen ableiten $[3,5,24]$, andere wiederum beobachteten, wie unsere Ergebnisse auch zeigen, eine Änderung der Diagnose ohne Beeinflussung der Therapie [10, 11, 19, 23, 26].

Eine Problemverletzung stellt die Condylus-radialis-Fraktur dar. Eine primäre Unterscheidung zwischen den stabilen inkompletten Brüchen, den so genannten „hängenden" Frakturen, und den instabilen vollständigen Frakturen, welche operativ behandelt werden müssen, gelingt mit der konventionellen Röntgenuntersuchung aufgrund der noch überwiegend knorpelig angelegten Epiphyse nicht. Die MRT ermöglicht eine gezielte Unterscheidung. Allerdings handelt es sich in der Regel um sehr junge Kinder mit einem Altersgipfel um das 4. Lebensjahr, sodass nicht selten eine Sedierung oder gar Narkose zur adäquaten Untersuchung notwendig sind. Gegen diese teilweise belastende und zeit- und kostenaufwändige Untersuchung spricht, dass in der Praxis eine ausreichend Unterscheidung zwischen stabilen und instabilen Frakturen erfolgen kann, indem nach 4-6 Tagen eine gipsfreie Röntgenkontrolluntersuchung zum Ausschluss einer Sekundärdislokation durchgeführt wird.

\section{Indikationen zur Durchführung einer}

MRT. Eine Indikation besteht für die MRT z. B. in der Darstellung begleitender Bandverletzungen nach der Ellenbogenluxation, welche vorwiegend im Adoleszentenalter auftritt ( Abb. 1). Eine weitere Indikation ergibt sich in der Diagnostik von Komplikationen nach Ellenbogenverletzung zur Darstellung von Osteonekrosen, osteochondralen Defekten oder freien Gelenkkörpern.

\section{Knie}

Dieses Gelenk ist in den letzten Jahren zur Domäne der MRT geworden. Sie wird allgemein zum Ausschluss eines Kniebinnenschadens beim unklaren posttraumatischen Knieschmerz empfohlen [8] und hat damit die rein diagnostische Arthroskopie in weiten Teilen abgelöst. Gerade beim Kind kann eine klinische Diagnose eines Kniebinnenschadens erschwert sein. Die Anamnese ist häufig nicht eruierbar, und eine klinische Untersuchung mit nachvollziehbaren Meniskus- und Stabilitätstests kann v. a. beim jungen Kind schwierig sein. Hinzu kommt eine erhöhte Bandlaxizität, welche eine Stabilitätsbeurteilung des Bandapparates in manchen Fällen erschwert. In diesen Fällen wird die Durchführung einer MRT auch im Kindesalter empfohlen. Dabei ist die Inzidenz von Kniebinnenschäden bei Kindern im Vergleich zum Erwachsenen deutlich geringer, was wahrscheinlich auf die höhere Flexibilität der osteochondralen sowie Bandstrukturen im Kindesalter zurückzuführen ist [35]. Allerdings nehmen die Sensitivität sowie die Spezifität der MRT bei Knieverletzungen mit sinkendem Alter ab [4, 20, 31]. Bei adoleszenten Kindern sind die Werte mit denen beim Erwachsenen vergleichbar. 
In der eigenen Untersuchung konnten in den MRT-Aufnahmen 4 zusätzliche Frakturen diagnostiziert werden. Dabei handelte es sich um subchondrale Impressionsfrakturen. Die Diagnose änderte sich in 11 Fällen, wobei dies im Wesentlichen auf das Vorliegen von Kniebinnenschäden wie Meniskusverletzung und Kreuzbandruptur oder auf osteochondrale Frakturen zurückzuführen war. Dies führte in 7 Fällen zu einer zusätzlichen Operationsindikation, die arthroskopisch realisiert wurde. Die Verletzung des Innenmeniskus war die häufigste Verletzung $(\mathrm{n}=10 ; 55 \%)$, hierbei war v. a. das Hinterhorn betroffen, gefolgt von der Außenmeniskusläsion $(n=6 ; 33 \%)$ und vorderen Kreuzbandruptur ( $n=6 ; 33 \%)$. In 12 Fällen konnte „bone bruise“ beobachtet werden, in 5 Fällen in Assoziation mit ligamentären Verletzungen. Lahm et al. [17] beobachteten in 210 MRT-Untersuchungen des Kniegelenks in 26 Fällen ein „bone bruise“, welches in 20 Fällen mit einer ligamentären Verletzung assoziiert war. In der Folgeuntersuchung 6 Wochen später konnte kein „bone bruise“ mehr nachgewiesen und somit kein prognostischer $\mathrm{Zu}$ sammenhang gesehen werden [17].

\section{Indikationen zur Durchführung einer}

MRT. Frakturen im Bereich des Kniegelenks können in aller Regel bereits ausreichend im konventionellen Röntgenbild beurteilt werden. Die MRT liefert bezüglich der akuten Frakturdiagnostik keine relevanten Zusatzinformationen. Allerdings kann sie gerade bei einem konservativen Vorgehen einen begleitenden Kniebinnenschaden ausschließen ( $($ Abb. 2). Eine wesentliche Rolle spielt sie zudem bei hemmenden Wachstumsstörungen nach kniegelenknahen Frakturen, insbesondere zur Darstellung der Knochenbrücke für die weitere Therapieplanung und Prognoseeinschätzung (• Abb. 3). Zudem hat sie in der Diagnostik nach $\mathrm{Pa}$ tellaluxationen eine zentrale Rolle inne. In unklaren Fällen sichert sie die Diagnose durch Darstellung des „bone bruise“ am lateralen Femurkondylus und medialen Patellarand. Sie stellt Begleitverletzungen, wie eine Ruptur des medialen Retinaculums oder des medialen patellofemoralen Ligaments (MPFL), sowie osteochondrale Defekte dar.

Trauma Berufskrankh 2010 - 12[Suppl 3]:329-334 DOI 10.1007/s10039-009-1569-z

C) Springer-Verlag 2010

\section{Schneidmueller $\cdot$ M. Maier $\cdot$ M. Mack · E. Geiger $\cdot$ I. Marzi Gelenkverletzungen im Kindes- und Jugendalter. Indikation und therapeutische Relevanz der MRT (Magnetresonanztomographie)}

\section{Zusammenfassung}

Die MRT (Magnetresonanztomographie) gewinnt in der Diagnostik von Verletzungen im Kindes- und Jugendalter zunehmend an Bedeutung, die therapeutische Relevanz der hiermit gewonnenen Zusatzinformationen wird kontrovers diskutiert. Neben den Einsatzmöglichkeiten der MRT bei Verletzungen im Kindes- und Jugendalter werden eigene Ergebnisse einer retrospektiven Studie präsentiert, bei welcher die posttraumatischen Röntgenbilder und MRT-Aufnahmen in insgesamt 45 Fällen von jeweils 2 Radiologen und 2 Unfallchirurgen anonymisiert voneinander unabhängig standardisiert ausgewertet sowie bezüglich ihrer therapeutischen Relevanz beurteilt wurden. Zusammenfassend ist festzustellen, dass die MRT weniger der Akutdiagnostik von Frakturen dient als vielmehr der
Verlaufsbeurteilung z. B. prolongierter Heilverläufe, um Komplikationen aufzudecken. Häufig wird sie zur Planung weiterer therapeutischer Schritte genutzt. Zudem gibt es spezielle Indikationen, bei der sie eine zentrale Rolle spielt, wie zum Ausschluss von Kniebinnenschäden, von Begleitverletzungen nach Patella- oder Schulterluxation, zur Stadieneinteilung der Osteochondrosis dissecans, zur Darstellung posttraumatischer Durchblutungsstörungen und zur Abgrenzung zu Differenzialdiagnosen.

\section{Schlüsselwörter}

Kindes- und Jugendalter - Gelenkverletzungen - Magnetresonanztomographie . Verlaufsbeurteilung $\cdot$ Therapieplanung

\section{Joint injury in childhood and adolescence. Indications and therapeutic relevance of MRI}

\section{Abstract}

MRI (magnetic resonance imaging) is becoming increasingly important in the diagnosis of injuries in children and adolescents. However, the therapeutic relevance of the additional information gained in this way is discussed controversially. In addition to detailing the possible uses of MRI in pediatric and adolescent injuries, results from retrospective studies are presented. These results include post-traumatic $X$-ray images and MRI images in a total of 45 cases, in each case taken by two radiologists and two trauma surgeons anonymously from each other, which were then evaluated in an independent and standardized fashion and assessed in terms of their therapeutic relevance. Looking at the data collectively, it becomes clear that MRI is more useful in evaluating injury course, e.g., prolonged healing, and identifying complications than it is in the acute diagnosis of fractures. It is often used in the planning of further therapeutic steps. In addition, there are special indications in which MRI plays a central role, such as excluding internal injuries of the knee joint, concomitant injuries following patella or shoulder luxation, staging of osteochondrosis dissecans, identifying post-traumatic circulation disorders and assisting in the differential diagnosis.

\section{Keywords}

Childhood and adolescence - Joint injury . Magnetic resonance imaging . Judging disease course $\cdot$ Therapy planning 

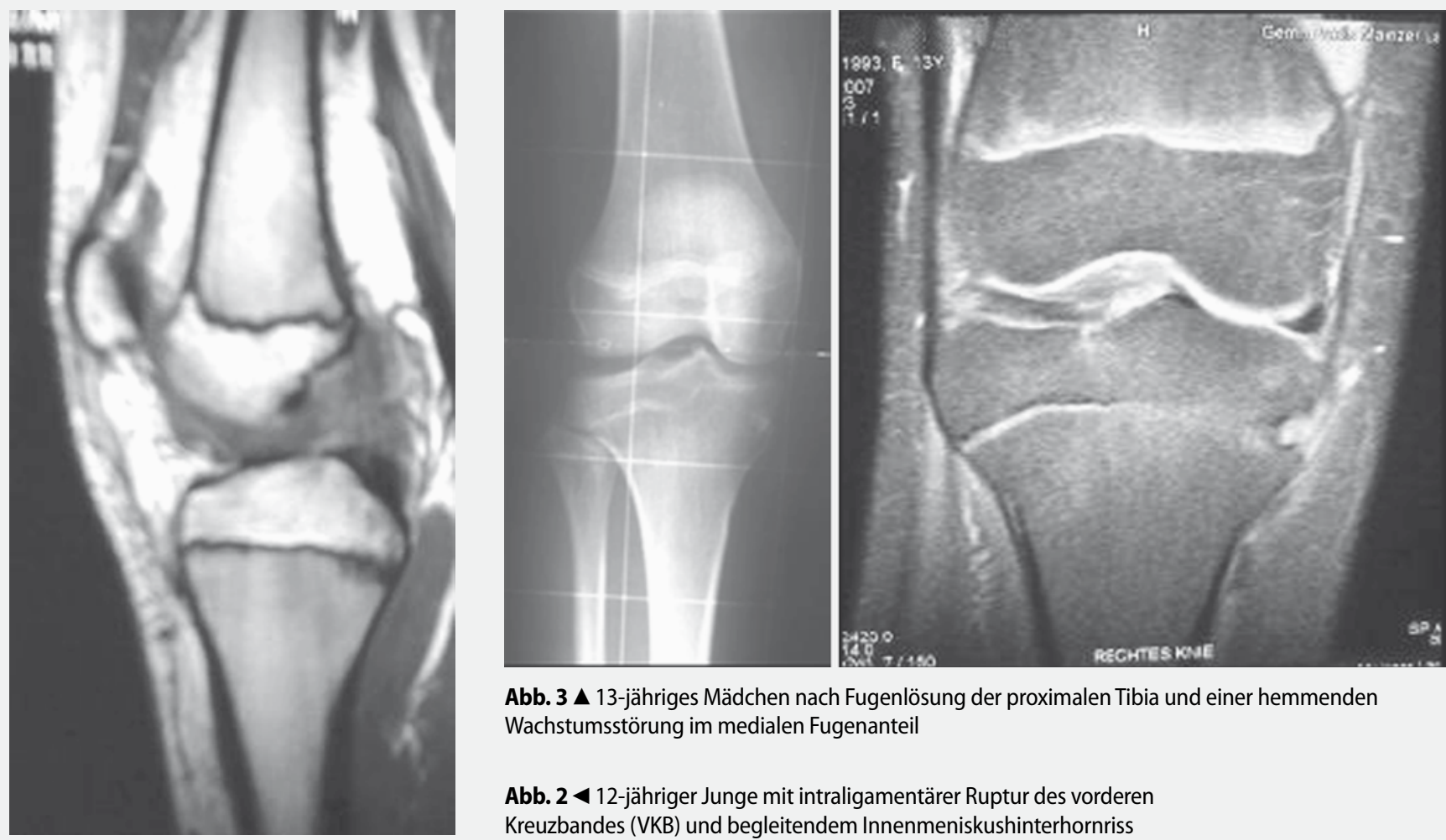

Abb. 3 \ 13-jähriges Mädchen nach Fugenlösung der proximalen Tibia und einer hemmenden Wachstumsstörung im medialen Fugenanteil

Abb. $2<12$-jähriger Junge mit intraligamentärer Ruptur des vorderen Kreuzbandes (VKB) und begleitendem Innenmeniskushinterhornriss
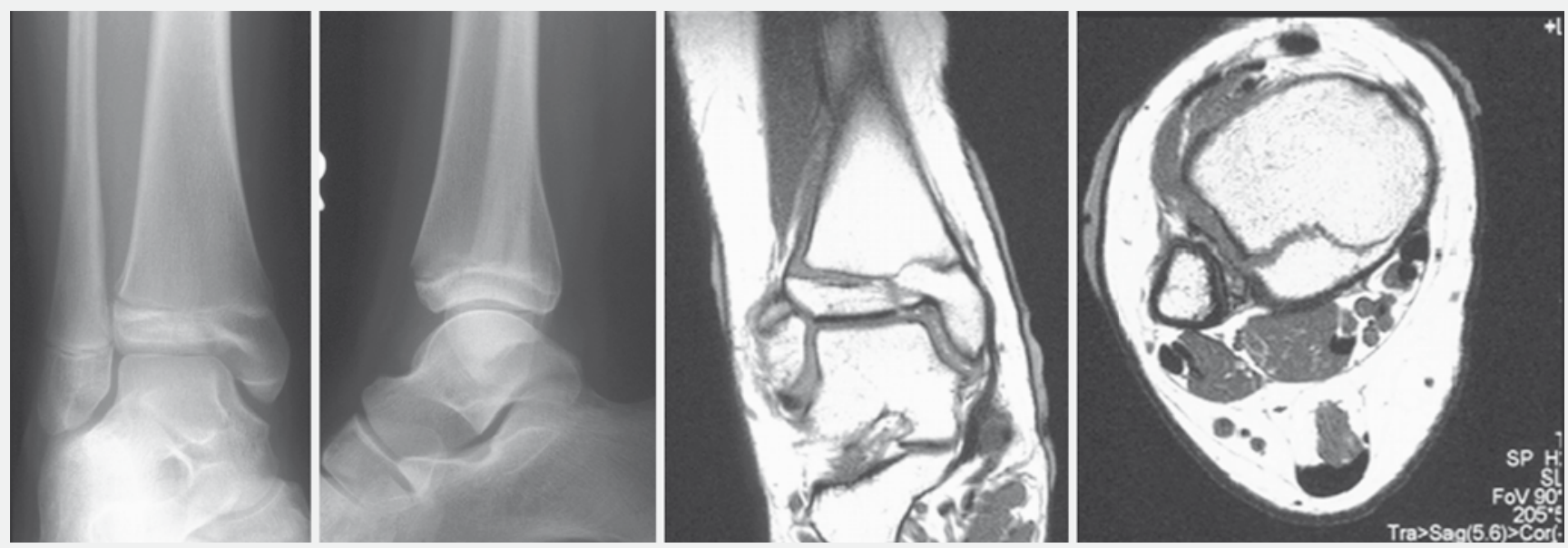

Abb. 4 \ "Triplane“-I-Fraktur bei einem 13-Jährigen, bereits im Röntgenbild sichtbar, MRT: adäquate Therapieplanung anhand des anschaulich dargestellten Frakturverlaufs möglich

\section{Oberes Sprunggelenk}

Sprunggelenkverletzungen sind im Kindesalter häufig. Die Zahl der erst beim Adoleszenten vorkommenden Übergangfrakturen liegt mit 6-10\% aller epiphysären Frakturen relativ hoch. Zur Wahl des optimalen therapeutischen Vorgehens ist eine genaue Diagnose notwendig. Doch gerade Übergangsfrakturen mit ihrem komplexen Frakturverlauf sind im konventionellen Röntgenbild insbesondere für Ungeübte häufig schwierig zu di- agnostizieren. Zur Zeit der Verbreitung der Computertomographie (CT) nahmen die Inzidenz der Übergangsfrakturen sowie auch der gestellten Operationsindikationen sprunghaft zu [27]. Es ist anzunehmen, dass dies nicht in einer plötzlich erhöhten Verletzungsanfälligkeit der Jugendlichen begründet war, sondern in der besseren Darstellung der Frakturen durch die CT. So wird von machen Autoren auch in der Diagnostik von Sprunggelenkfrakturen die MRT empfohlen [5, 14, 27].
In unserer Studie konnten insgesamt 20 Sprunggelenke untersucht werden. Dabei änderte sich die Diagnose in 12 Fällen. In immerhin 8 dieser Fälle kam es zu einer Änderung der Frakturklassifikation, eine zusätzliche Fraktur wurde diagnostiziert, ein primärer Frakturverdacht wurde ausgeschlossen, bei 2 in der Röntgenaufnahme unauffälligen Sprunggelenken konnten in der MRT Verletzungen des Bandapparates nachgewiesen werden. Insgesamt konnten in 9 Fällen Bandverletzungen und in 18 Fällen ein „bone bruise“ gese- 
hen werden. Eine Änderung des therapeutischen Vorgehens war innerhalb des konservativen Bereichs (funktionell vs. Immobilisation) in 6 Fällen erforderlich, eine zusätzliche Operationsindikation ergab sich nicht.

Aufgrund der fehlenden Strahlenbelastung und der guten Möglichkeit, auch Band- und Syndesmosenverletzungen sowie Osteonekrosen, wie sie nicht selten im Bereich des Talus posttraumatisch auftreten, darzustellen, steht die MRT auch im Bereich des Sprunggelenks in der erweiterten Diagnostik im Vordergrund. Sie wird deshalb bei persistierenden Schmerzen posttraumatisch zur weiteren Abklärung empfohlen.

In bisherigen Untersuchungen wurde eine unterschiedliche Wertigkeit der MRT nach akutem Sprunggelenktrauma beobachtet. Seifert et al.[27] konnten an 15 Sprunggelenkfrakturen 2-mal eine Änderung der Frakturklassifikation sowie eine Unterschätzung der Frakturdislokation um durchschnittlich 0,5 $\mathrm{mm}$ beobachten. In einem Fall ergab sich eine zusätzliche Operationsindikation. Lohman et al. [18] beobachteten 2001 an 60 Sprunggelenken in $21 \%$ eine Änderung der Diagnose jeweils ohne therapeutische Konsequenz. Auch Petit et al. [23] verzeichneten bei 29 Sprunggelenken in nur einem Fall eine Diagnoseänderung, ebenfalls ohne therapeutische Konsequenz. IwinskaZelder et al. [14] dagegen beschrieben bei 1o Kindern in 8 Fällen eine Änderung der Diagnose, was in 4 Fällen zu einem Therapiewechsel führte, und Herber et al. [13] diagnostizierten okkulte Frakturen in 11 von 28 Kindern mit einer Therapieänderung in 35 von 55 Kindern.

„bone bruise“. In unserem Patientenkollektiv konnte „bone bruise“ in 18 von 21 Fällen $(86 \%)$ gesehen werden, wobei Tibia, Fibula und der Talus gleichmäßig häufig betroffen waren. Lohman et al. [18] fanden eine Häufigkeit von $64 \%$ ohne akute therapeutische Relevanz. Zanetti et al. [33] zeigten, dass "bone bruise“ sowie auch in der MRT sichtbare Bandverletzungen nicht mit der Prognose einer Sprunggelenkverletzung zusammenhängen. Alanen et al. [1] fanden eine „bone bruise"-Inzidenz von $27 \%$ nach Distorsionstrauma ohne Auswirkungen auf die klinischen Ergebnisse. Eine weitere Arbeit zeigte das Auftreten von „bone bruise“ in $57 \%$ völlig asymptomatischer kindlicher Füße, was als normale Wachstumserscheinung interpretiert wurde und nicht zwangsläufig als pathologisches Zeichen [22].

Indikationen zur Durchführung einer MRT. Trotz häufig fehlender therapeutischer Konsequenz kann die MRT in nicht eindeutigen Fällen zur Sicherung der Diagnose indiziert sein, sodass wir sie bei unklaren Schmerzen, die länger als 1 Woche andauern, empfehlen. Zudem vereinfacht sie eine Operationsplanung durch dreidimensionale Darstellung des Frakturverlaufs speziell bei Übergangsfrakturen (- Abb. 4; [4]). Eine weitere Indikation ergibt sich in der Darstellung von posttraumatischen Wachstumsstörungen, um das Ausmaß und die Lokalisation für eine weitere Therapie genau darstellen zu können. Eine besondere Rolle spielt die MRT in der Diagnostik und Stadienbeurteilung der Osteochondrosis dissecans, nach der sich die weitere Therapie richtet [6].

\section{Weitere Lokalisationen und Indikationen}

Schulter. Am Schultergelenk spielt die MRT nach Schulterluxation zum Ausschluss einer Labrumverletzung eine große Rolle. Unbehandelt führt diese zu einer erhöhten Reluxationsrate [28]. Allerdings ist die Schulterluxation eher eine Verletzung des jungen Erwachsenen und nur sehr selten bei Kindern zu finden [7].

Hand und Fuß. Vor allem im Bereich der Hand- oder Fußwurzel ermöglicht die MRT die Diagnostik von okkulten Frakturen. Bandverletzungen oder Verletzungen des TFCC (triangulärer fibrokartilaginärer Komplex), wie wir sie nicht selten als Ursache für Handgelenkschmerzen bei Erwachsenen finden, sind im Kindesalter eine Rarität [2].

Weitere Verletzungen. Eine weitere Möglichkeit besteht in der Darstellung von Überlastungsschäden und Stressreaktionen bis hin zur Stressfraktur v. a. bei sportlich aktiven Kindern. Neben einer
Stadieneinteilung kann eine Abgrenzung zu Differenzialdiagnosen wie einer Osteomyelitis oder einem osteogenen Sarkom erfolgen [9].

In unklaren Fällen kann die MRT eine Abgrenzung zu so genannten orthopädischen Krankheitsbildern liefern. Insbesondere am Hüftgelenk kann sie bei unklaren Schmerzen und unauffälligem Röntgenbild z. B. eine Frühdiagnose des Morbus Perthes, der Epiphyseolysis capitis femoris oder einer Osteomyelitis ermöglichen. Auch in der Beurteilung der Hüftkopfdurchblutung im Verlauf einer Epiphyseolyse oder einer Schenkelhalsfraktur spielt sie eine besondere Rolle. Sie kann Ausdehnung und Lage einer Kopfnekrose darstellen, um ggf. weitere Schritte wie eine Umstellungsosteotomie planen zu können.

\section{Fazit}

Die MRT ermöglicht die Darstellung okkulter Frakturen und kann v. a. komplexe Frakturverläufe in unklaren Fällen verbessert darstellen. Allerdings spielt sie in der Akutdiagnostik von Frakturen weniger eine Rolle als vielmehr in der Verlaufsbeurteilung von prolongierten Heilverläufen. Hierbei können z. B. Wachstumsstörungen, Osteonekrosen, freie Gelenkkörper oder auch Knorpeldefekte aufgedeckt werden. Zudem hat sie bei speziellen Indikationen eine zentrale Rolle inne, wie in der Diagnostik eines Kniebinnenschadens, zum Ausschluss von Begleitverletzungen nach Patella- oder Schulterluxation, zur Diagnose und Stadieneinteilung einer Osteochondrosis dissecans oder Stressreaktionen, und ermöglicht zudem eine Abgrenzung zu möglichen Differenzialdiagnosen wie Tumor oder Osteomyelitis.

\section{Korrespondenzadresse}

Dr. D. Schneidmueller

Abteilung für Unfall-, Hand-

und Wiederherstellungschirurgie,

Johann-Wolfgang-Goethe-Universität Frankfurt, Theodor-Stern-Kai 7, 60590 Frankfurt am Main dorien.schneidmueller@kgu.de

Interessenkonflikt. Der korrespondierende Autor gibt an, dass kein Interessenkonflikt besteht. 


\section{Literatur}

1. Alanen V, Taimela S, Kinnunen J et al (1998) Incidence and clinical significance of bone bruise after supination injury of the ankle. J Bone Joint Surg Br 80:513-515

2. Alt V, Gasnier J, Sicre G (2004) Injuries of the scapholunate ligament in children. J Pediatr Orthop B 13:326-329

3. Beltran J, Rosenberg ZS, Kawelblum M et al (1994) Pediatric elbow fractures: MRI evaluation. Skeletal Radiol 23:277-281

4. Borsa JJ, Peterson HA, Ehman RL (1996) MR imaging of physeal bars. Radiology 199:683-678

5. Carey J, Spence L, Blickman H, Eustace S (1998) MRI of pediatric growth plate injury: correlation with plain film radiographs and clinical outcome. Skeletal Radiol 27:250-255

6. Ecklund K (2002) Magnetic resonance imaging of pediatric musculoskeletal trauma. Top Magn Reson Imaging 13:203-218

7. Emery KH (2009) MR imaging in congenital and acquired disorders of the pediatric upper extremity. Magn Reson Imaging Clin N Am 17:549-570

8. Fischer SP, Fox JM, Del Pizzo W et al (1991) Accuracy of diagnoses from magnetic resonance imaging of the knee. J Bone Joint Surg Am 73:2-10

9. Fredericson M, Bergman AG, Hoffman KL, Dillingham MS (1995) Tibial stress reaction in runners. Correlation of clinical symptoms and scintigraphy with a new magnetic resonance imaging grading system. Am J Sports Med 23:472-481

10. Gordon AC, Friedman L, White PG (1997) Pictorial review: magnetic resonance imaging of the paediatric elbow. Clin Radiol 52:582-588

11. Griffith JF, Roebuck DJ, Cheng JC et al (2001) Acute elbow trauma in children: spectrum of injury revealed by MR imaging not apparent on radiographs. AJR Am J Roentgenol 176:53-60

12. Halsted MJ, Kumar H, Paquin JJ et al (2004) Diagnostic errors by radiology residents in interpreting pediatric radiographs in an emergency setting. Pediatr Radiol 34:331-336

13. Herber S, Kreitner KF, Kalden P et al (2000) Low field MRI of ankle joint: initial experience in children and adolescents using an open 0.2 T MR-system. Rofo Fortschr Geb Rontgenstr Neuen Bildgeb Verfahr 172:267-273

14. Iwinska-Zelder J, Schmidt S, Ishaque N et al (1999) Epiphysenfugenverletzungen der distalen Tibia. Sinnvolle Mehrinformation durch die MRT? Radiologe 39:25-29

15. Jaramillo D, Hoffer FA, Shapiro F, Rand F (1990) MR imaging of fractures of the growth plate. AJR Am J Roentgenol 155:1261-1265

16. Kan JH, Estrada C, Hasan U et al (2009) Management of occult fractures in the skeletally immature patient: cost analysis of implementing a limited trauma magnetic resonance imaging protocol. Pediatr Emerg Care 25:226-230

17. Lahm A, Erggelet $C$, Steinwachs M, Reichelt A (1998) Articular and osseous lesions in recent ligament tears: arthroscopic changes compared with magnetic resonance imaging findings. Arthroscopy $14: 597-604$

18. Lohman M, Kivisaari A, Kallio P et al (2001) Acute peadiatric ankle trauma: $M R I$ versus plain radiography. Skeletal Radiol 30:504-511

19. Major NM, Crawford ST (2002) Elbow effusions in trauma in adults and children: is there an occult fracture? AJR Am J Roentgenol 178:413-418
20. McDermott MJ, Bathgate B, Gillingham BL, Hennrikus WL (1998) Correlation of MRI and arthroscopic diagnosis of the knee pathology in children and adolescent. J Pediatr Orthop 18:675-678

21. Naranja RJ, Gregg JR, Dormans JP et al (1997) Pediatric fracture without radiographic abnormality: description and significance. Clin Orthop 1:141146

22. Pal CR, Tasker AD, Ostlere SJ, Watson MS (1999) Heterogeneous signal in bone marrow on MRI of children's feet: a normal finding? Skeletal Radiol 28:274-278

23. Petit $P$, Panuel M, Faure F et al (1996) Acute fracture of the distal tibial physis: role of gradientecho MR imaging versus plain film examination. AJR Am J Roentgenol 166:1203-1206

24. Pudas T, Hurme T, Mattila K, Svedström E (2005) Magnetic resonance imaging in pediatric elbow fractures. Acta Radiol 46:636-644

25. Rivara FP, Parish RA, Mueller BA (1986) Extremity injuries in children: predictive value of clinical findings. Pediatrics 78:803-807

26. Schneidmueller D, Maier M, Mack M et al (2005) Therapeutic relevance of magnetic resonance imaging in joint injuries in children. Unfallchirurg 108:537-543

27. Seifert J, Laun R, Paris S et al (2001) Die Wertigkeit der Magnetresonanztomografie (MRT) bei der Diagnostik von Übergangsfrakturen im Bereich der distalen Tibia. Unfallchirurg 104:524-529

28. Simonet WT, Cofield RH (1984) Prognosis in anterior shoulder dislocation. Am J Sports Med 12:19-24

29. Skaggs DL (1997) Elbow fractures in children: diagnosis and management. J Am Acad Orthop Surg 5:303-312

30. Skaggs D, Pershad J (1997) Pediatric elbow trauma. Pediatr Emerg Care 13:425-434

31. Stanitski CL (1998) Correlation of arthroscopic and clinical examinations with magnetic resonance imaging findings of injured knees in children and adolescents. Am J Sports Med 26:2-6

32. Sugimoto H, Ohsawa T (1994) Ulnar collateral ligament in the growing elbow: MR imaging of normal development and throwing injuries. Radiology 192:417-422

33. Zanetti M, De Simoni C, Wetz HH et al (1997) Magnetic resonance imaging of injuries to ankle joint: can it predict clinical outcome? Skeletal Radiol 26:82-88

34. Zitzewitz H von, Kreitner KF, Eble F, Thelen M (1998) Kindliche Frakturen - Nutzen der Kernspintomographie für Diagnose und Einteilung. RoFo 168:52

35. Zobel MS, Borrello JA, Siegel MJ, Steward NR (1994) Pediatric knee MR imaging: pattern of injuries in the immature skeleton. Radiology 190:397401 